\title{
Research on the Evaluation of Service Status of Tunnel Structure Near the Station
}

\author{
$\mathrm{Z} \mathrm{J} \mathrm{LIN}^{\mathrm{a}}, \mathrm{S} \mathrm{GAO}^{\mathrm{b}}, \mathrm{J} \mathrm{LI}^{\mathrm{c}}$ and $\mathrm{J} \mathrm{C} \mathrm{WANG}^{\mathrm{c}, 1}$ \\ ${ }^{a}$ Transportation Institute, Hydrochina Huadong Engineering Corp., Hangzhou, \\ Zhejiang, China \\ ${ }^{\mathrm{b}}$ School of Civil Engineering, Shenyang Jianzhu University, Shenyang, Liaoning, \\ China \\ ' Institute of Transportation Engineering, Zhejiang University, Hangzhou, Zhejiang, \\ China
}

\begin{abstract}
To study the service status of the tunnel structure near the station, a disease detection of Metro Line 1 near the station during the operation period was carried out. The four major tunnel structural diseases including water leakage, segment stagger, open joints, and lining cracks were counted. The distribution law of the structural diseases along the longitudinal and circumferential directions of the interval tunnel was analyzed. The service status of segment components and connecting bolts were evaluated based on existing specification. The overall service status of the tunnel was determined. The service status level of the upstream line was determined as "iii", and the service status was "degradation", the service status level of the downstream line was determined as "iv" and the service status was "deteriorated".
\end{abstract}

Keywords. Tunnel, service status, structural diseases

\section{Introduction}

The service status of subway tunnels is an important factor that affects the economic development and daily travel safety. Scholars have studied the service status of tunnels. The bearing capacity of the tunnel decreases with the peeling of the lining structure [1]. The reason of the leakage of a tunnel was summarized based on the experience of the tunnel seepage engineering, and it was concluded that the leakage of the tunnel was directly or indirectly affected by the natural conditions, the engineering geological conditions, the design and construction, and the transportation of the tunnel [2]. The damage status, crack development degree and distribution law of the segment were calculated based on the large number of segment cracking and falling off during the construction of the shield tunnel. The damage characteristics of the segment structure were analyzed under the action of various construction influencing factors [3]. The bearing capacity of the lining changes due to the existence of cracks, and the external construction easily lead to the continued development of existing cracks and the generation of new cracks [4]. The main causes of the structural water leakage, segment cracking, open joints, and excessive tunnel deformation were explored through the fault

\footnotetext{
${ }^{1} \mathrm{~J}$ C Wang, Institute of Transportation Engineering, Zhejiang University, Hangzhou, Zhejiang, China; E-mail: wjc501@zju.edu.cn.
} 
model tree, which are divided into three unfavorable factors in the pre-design, the construction and the post-operation management [5]. The existence of cracks will cause a certain attenuation of the overall stiffness of the segment, which has been studied by similar model tests [6].

In this paper, structural diseases including water leakage, segment stagger, open joints, and lining cracks were counted. The distribution law of structural diseases along the longitudinal and circumferential directions of the interval tunnel was analyzed. The service status of segment components and connecting bolts were evaluated based on existing specification, the overall service status of the tunnel was determined.

\section{Project Overview}

The tunnel is located in a typical soft soil area, with an inner diameter of $5.5 \mathrm{~m}$, an outer diameter of $6.2 \mathrm{~m}$, and a ring width of $1.2 \mathrm{~m}$. The single-ring tunnel consists of 6 concrete grade C50 segments connected by M30 bending bolts. The mileage number of the interval tunnel is $\mathrm{K} 15+621.880-\mathrm{K} 15+813.877$, of which the up line is from ring 475 to ring 315 , the 475 ring is the last ring of the up line from the station tunnel, the down line is from Ring 1 to Ring 160, and the first ring is the starting ring where the down line exits from the station.

\section{Tunnel Disease Statistics}

\subsection{Water Leakage}

Tunnel leakage diseases include wet spots, water seepage, dripping, and some areas with more serious leakage and even sediment seepage. The leakage diseases of the tunnel are mainly concentrated in the joints, and the degree of leakage of the pipe segment structure is relatively weak.

\subsection{Segment Stagger}

The relevant regulations on the dislocation between the segments of the tunnel and the dislocation within the ring are made by the "Code for Construction and Acceptance of Shield Tunnelling Method" (GB50446-2017) [7]. The allowable deviation of the segment stagger between rings is $15 \mathrm{~mm}$, and the allowable deviation of segment stagger in the ring is $10 \mathrm{~mm}$. The maximum segment stagger does not exceed $15 \mathrm{~mm}$, which meets the specification for segment stagger between rings. The maximum segment stagger of the up-line is $10 \mathrm{~mm}$; the maximum segment stagger of the downline is $10.5 \mathrm{~mm}$, which exceeds the specification requirements.

\subsection{Lining Cracks}

The cracks on the up-line are concentrated between the 440th ring and the 330th ring, totaling 308; the cracks on the down-line are concentrated between the 35th ring and the 145 th ring, totaling 497 . The cracks are concentrated on the lining vault, and the crack depths of the up-line and the down-line are relatively large. 


\subsection{Open Joints}

According to the "Code for Structural Appraisal of Shield Tunnel" (DGTJ-08-21232013) [8], the allowable opening amount is $6 \mathrm{~mm}$. The opening amount of the longitudinal joints of all the up-line segments is between 1-2 times of the allowable opening amount, there are 4 rings where the maximum longitudinal joints opening reaches $11 \mathrm{~mm}$, and the joint opening phenomenon occurs at the first ring segment that connects with the station, and the opening amount reaches $10 \mathrm{~mm}$; There is only one ring meeting the allowable value, 7 rings being $1 \sim 2$ times of the allowable opening amount, 6 rings being 2 3 times of the allowable opening amount, the maximum opening amount reaches $20 \mathrm{~mm}$, which is greater than 3 times of the allowable opening amount.

\section{Analysis on the Distribution Law of Tunnel Diseases}

\subsection{Distribution Law of Tunnel Diseases along the Longitudinal Direction of the Tunnel}

The distribution law of segment cracks along the longitudinal direction of the tunnel is consistent with the distribution law of convergence. The convergence between the near station tunnel and the remote tunnel is small, and there is no crack disease phenomenon, while near the extreme point of tunnel convergence, the tunnel crack disease phenomenon concentrated. Structural connectivity diseases such as water leakage, segment stagger, and joint openings occur throughout the tunnels. However, the structural connectivity diseases are more serious near the extreme points of tunnel convergence.

\subsection{Distribution Law of Tunnel Structural Diseases along the Ring Direction}

According to the statistical analysis of the previous data, the leakage diseases of the tunnel structure mainly occur at the joints of the pipe segments, and the leakage diseases at the joints are mainly concentrated in the ring joints. The staggered positions of the largest inter-ring segments of the tunnel all appear at the arch waist position. The maximum segment stagger in a ring is mainly concentrated on the tunnel vault. The maximum opening of the longitudinal joint of the segment is mainly concentrated on the vault. The tunnel lining cracks near the maximum convergence are basically longitudinal cracks, which are all distributed at the position of the lining vault.

\section{Evaluation of Tunnel Structure Status}

The structural service status evaluation of the tunnel includes two parts: the segment structure and the segment connection service status evaluation. According to the "Code for Structural Appraisal of Shield Tunnel" (DGTJ-08-2123-2013) [8], the service status of the interval tunnels in the affected area was classified, and the structural damage of the tunnel was evaluated. 


\subsection{Service Status Evaluation of Segment Structure}

According to the geological survey data, the chloride ion concentration in the groundwater near the site is less than $100 \mathrm{mg} / \mathrm{L}$, The environment where the tunnel located is relatively humid. Based on the requirements of the specification [8], the environmental action level is defined as Class B, and the service status of the components is divided according to the cracks and water leakage of the pipe segment. The service status of each ring segment of the tunnel is shown in figure 1.

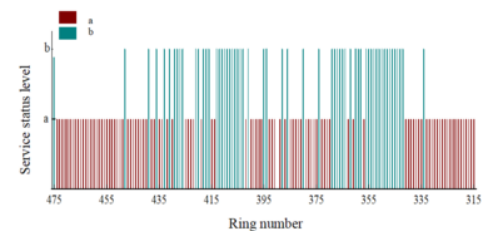

Up-line

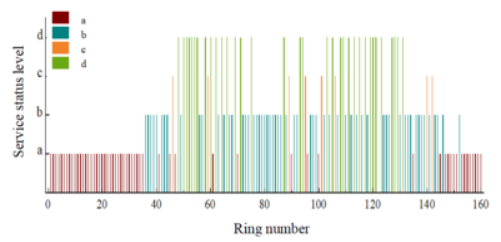

Down-line

Figure 1. Service status evaluation of segment structure.

\subsection{Evaluation of the Service State of the Segment Structure Connection}

According to the water leakage at the joints of the pipes, the opening of the joints, and the misalignment of the pipes, the service status of the connection of the pipes is classified [8]. The service status of the segment connections is shown in figure 2. Compared with the up-line, the segment connections of the tunnels in the down-line are in poorer service status, mostly at level d.

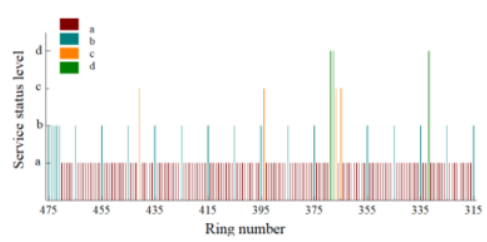

Up-line

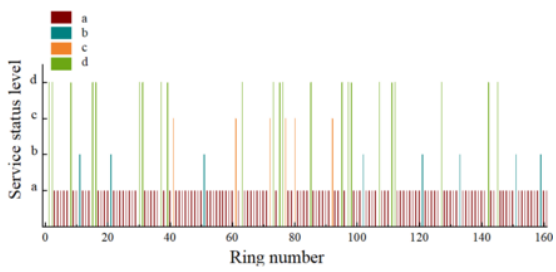

Down-line

Figure 2. Evaluation of the service state of the segment structure connection.

\subsection{Evaluation of Tunnel Service Status}

Based on the above evaluation of the service status of the tunnel segment structure and the structural connection above, the overall service status of the tunnel was determined.

(1) The service status of up-line components is rated as "a" and "b", the service status of component connections was rated as "a", "b", "c", "d", and the proportion of " $\mathrm{d}$ " components and connections was less than $10 \%$. Based on the requirements of the specification, the service status of the up-line was determined to be "iii" and the service status was "degradation". It was believed that the function of the tunnel was impaired and cannot be used normally.

(2) The service status of down-line components was rated as "a", "b", "c", "d", the service status of component connections was rated as "a", "b", "c", "d", and the proportion of " $\mathrm{d}$ " components and connections was more than $10 \%$. Based on the 
requirements of the specification, the service status of the down-line was determined to be "iv" and the service status was "deteriorated". It was believed that the applicability of the tunnel was affected, but the safe operation of the tunnel will not be affected.

\section{Conclusions}

(1) Tunnel structural diseases including water leakage, segment stagger, open joints, and lining cracks occur in a tunnel near the station. The structural disease of the downline tunnel is more serious than that of the up-line, and there are many sediment seepages in the ring joints.

(2) The distribution law of segment cracks along the longitudinal direction of the tunnel is consistent with the distribution law of convergence. The convergence between the near station tunnel and the remote tunnel is small, and there is no crack disease while near the extreme point of tunnel convergence, the tunnel crack disease concentrated. Structural connectivity diseases such as water leakage, segment stagger, and joint openings occur throughout the tunnels. However, the structural connectivity diseases are more serious near the extreme points of tunnel convergence.

(3) The maximum segment stagger in the segment ring, the maximum joint opening, and lining cracks are mainly concentrated in the position of the tunnel vault.

(4) The service status level of the upstream line was determined as "iii", and the service status was "degradation", the service status level of the downstream line was determined as "iv" and the service status was "deteriorated".

\section{References}

[1] Wang S, Liu C, Ma G. Experimental investigation on the influence of regional concrete spalling on shield tunnel segments. Advances in Civil Engineering. 2019 Jun; 2019(10): 1-15.

[2] Zhai ZP. Simple analysis on disease causes of tunnel water seepage. Chinese Journal of Underground Space and Engineering. 2017 Apr; 13(S1): 446-452.

[3] Lu DY, Sun WH, Sun A, He C. Statistics and analysis of cracking disease in segment lining for shield tunnel in construction stage. Journal of Railway Engineering Society. 2018 Jun; 35(06): 59-66+87.

[4] He ZY, Zhong HW and Chen ZH. Safety evaluation and finite element analysis of tunnel lining with cracks. Tunnel Construction. 2019 Dec; 39(S2): 69-77.

[5] Huang C, Chen WJ, Sun D and Lei GP. Analysis of causes of structural diseases in metro tunnels and summary of fault tree models. Modern Tunnelling Technology. 2020 Nov; 57(S1): 264-272.

[6] Wang S, Liu C, Shao Z, et al. Experimental study on damage evolution characteristics of segment structure of shield tunnel with cracks based on acoustic emission information. Engineering Failure Analysis. 2020 Dec; 118: 104899.

[7] GB50446-2017-2017. Code for construction and acceptance of shield tunnelling method. China Architecture \& Building Press, Beijing, China; 2017.

[8] DGTJ-08-2123-2013. Code for structural appraisal of shield tunnel. Shanghai: Tongji University; 2013. 